\title{
The ubiquitous weekend effect: moving past proving it exists to clarifying what causes it
}

\section{Richard J Lilford, Yen-Fu Chen}

Warwick Centre for Applied Health Research \& Delivery, Division of Health Sciences, University of Warwick, Coventry, UK

\section{Correspondence to} Professor Richard J Lilford, Room A154, 1st Floor, Division of Health Sciences, Warwick Medical School, University of Warwick, Coventry CV4 7AL, UK; r.j.lilford@warwick.ac.uk

Accepted 4 May 2015

\section{SLinked}

- http://dx.doi.org/10.1136/ bmjqs-2014-003467

\section{CrossMark}

\footnotetext{
To cite: Lilford RJ, Chen Y-F. BMJ Qual Saf 2015;24: 480-482.
}

Since the publication of the influential paper by Bell and Redelmeier in 2001, the 'weekend effect', whereby patients admitted to hospital over the weekend experience worse outcomes compared with apparently similar patients admitted during weekdays, has been explored in at least 105 studies. $^{2-6}$ In this issue, Ruiz et $\mathrm{al}^{7}$ document such an effect across four countries (Australia, England, Netherlands, and the USA) that contribute to the Global Comparators Project ${ }^{8}$ where electronic summary data are combined across borders. Twenty-eight metropolitan city hospitals contributed data from nearly 3 million admissions. The ubiquitous weekend effect is replicated yet again among emergency admissions in all four countries for hospital mortality measured at 7 days and, except for Australia, at 30 days. For elective surgical procedures, a weekend effect on mortality was observed in all four countries and additionally a 'Friday effect' was seen in Dutch hospitals included in the study. The findings are interesting, but should we all rush to Australia to see how acute medical services should be organised so as to avoid a high 30-day mortality rate associated with emergency admissions?

Hospitals included in this study constitute only a very small proportion of all hospitals in respective countries. The data are unlikely to be representative and consequently drawing any conclusion with regard to international comparison could be misleading. The existence of weekend mortality effects within 7 days following admission has been demonstrated previously in a larger study covering $>500$ hospitals in Australia. ${ }^{9}$ Failure to confirm the 7-day weekend mortality hike at 30 days in the current study can plausibly be ascribed to a diminished signal-to-noise ratio; as time passes new and recurrent illness supervenes, diluting any effect from the admission itself. So, taken in the round, the paper confirms that the weekend effect is a common finding at the hospital-wide level. Replicating this further would seem to fulfil no useful purpose. The question is what causes the weekend effect. Understanding the weekend effect is an extremely important task since it is large at about $10 \%$ in relative risk terms and $0.4 \%$ in percentage point terms. This amounts to about 160 additional deaths in a hospital with 40000 discharges per year. But how much of the observed increase results from service failure? And here is the rub, for while a 0.4 percentage point represents a large, potentially scandalous, number of deaths, it is quite a small proportional changes. Working out the proportions of these deaths that result from avoidable and unavoidable factors is thus no easy task. So how may the issue of weekend effect be further explicated when we move from whether the weekend effect exists to why?

1. More detailed analyses of databases: Candidate explanations for the weekend effect consist of differences in case-mix and service quality. ${ }^{10}$ In all four countries in the current study, more patients were admitted on weekdays than at weekendsabout $20 \%$ more. It follows that pathways to admission differ, with a higher proportion of patients admitted through the emergency department over the weekend. ${ }^{11}$ Patients admitted at weekends may be sicker and clinically more unstable. $^{12}$ Unfortunately, data held on routine databases such as those used in current study are often limited in quality and detail to enable the necessary statistical adjustment to be made. This limitation can be mitigated by data collected from clinical records and registries. For example, a single-centre study from 
Dublin showed that the overall weekend effect weakened when risk adjustment was made over a richer set of data that included physiological measurements at admission, ${ }^{13}$ whereas data from the US Myocardial Infarction Data Acquisition System (MIDAS) demonstrated a robust weekend effect on mortality having adjusted for clinical features such as type and site of infarction. ${ }^{14}$ However, risk adjustment can also obscure a genuine effect when care quality declines in line with increasing risk. In short, risk adjustment is a blunt tool, particularly when, as in the case of hospital mortality, most deaths are not preventable. ${ }^{15}$ Analyses of large datasets may provide clues to the causes of the weekend effect, but the issue of causality can never be resolved by purely 'desktop studies'.

2. Direct examination of quality of care during weekdays and over weekends: There are a priori reasons to suspect suboptimal standards of care during weekends since staffing ratios (especially for medical staff) and access to diagnostic services are reduced. ${ }^{16}$ Quantitative and qualitative observations of the quality of clinical care at weekend have shown that clinical processes are affected over weekends with higher error rates ${ }^{17}$ including longer delays, ${ }^{2} 6$ lower likelihood of receiving effective interventions $^{14}$ and higher rates of preventable adverse events. $^{18}$

3. Disease-specific studies: Previous studies have shown that the weekend effect does not apply to all diagnoses. ${ }^{1} 29$ There are both theoretical grounds and empirical evidence to suggest that weekend effect on mortality is more likely to occur in conditions such as cardiac arrhythmia and pulmonary embolism, ${ }^{19}$ where there is a high risk of death immediately after the onset of clinical events, during which timely interventions can reduce the risk. The increasing availability of routine databases covering large populations will allow more in-depth analyses by individual diagnoses in the future. Good examples include the aforementioned myocardial infarction study based on MIDAS, ${ }^{14}$ which evaluated the association between measures of quality of care and disease-specific outcomes; and the Australian study by Perez Concha and colleagues, in which temporal patterns (time-course) of the weekend effect for different diagnoses were studied in detail to elucidate possible causes. ${ }^{9}$ One potential caveat for examining disease-specific outcomes is that it depends on accurate diagnosis, but one of the putative reasons for weekend effect is that people might not be given correct diagnosis at weekends.

4. Intervention studies: Finding further evidence of the weekend effect and the necessary actions to mitigate it (where exists) requires appropriate evaluation studies alongside the introduction of an intervention. The English National Health Service is following a policy of incentivising hospitals to increase consultant cover over weekends. This policy is being evaluated by means of a study to track the effects of the intervention. ${ }^{19}$ The weekend effect will be compared over time in all hospitals in England (a difference in difference-type approach - the difference in mortality between weekend and weekday admissions over different time periods). Difference in rates at which hospitals acquire extra staff will also be exploited (a difference in difference in difference type of approach). English hospitals will also be compared with the other three countries of the UK that are not pursuing the policy. The study will also examine the differences in mortality according to pathways by which patients gain emergency admission (eg, A\&E vs outpatient department). Lastly, in-depth observations of the quality of care will be made from subsets of hospitals using mixed methods approaches, ${ }^{20}$ based on ethnography and case note review. Of course, this is not a randomised comparison and therefore falls short of the kind of proof that would be usual in clinical research. Moreover, there are many other changes taking place in the service that might affect the findings. Nevertheless, by triangulating data of different types by means of a formal model and including expert opinion informed by the literature, ${ }^{21}$ the study hopes to offer deeper insights than statistical analyses of routine data are able to provide.

Ruiz et al have exploited a truly novel database, and international comparisons always make for compelling reading. Readers of the journal will enjoy the paper, which shows that the weekend effect that has persisted over time also persists across space. However, attention should now turn to in-depth studies that can shed some light on the cause of weekend effect or on how it might be mitigated.

Contributors Both authors contributed to drafting and revising the manuscript.

Funding The authors have been funded by the National Institute for Health Research (NIHR) Collaboration for Leadership in Applied Health Research and Care (CLAHRC) West Midlands and the NIHR Health Services and Delivery Research Programme through the High-Intensity Specialist-Led Acute Care (HiSLAC) project.

Disclaimer The views expressed here are those of the authors and not necessarily those of the NIHR or Department of Health or NHS.

Competing interests Both authors are named investigators in the English High-Intensity Specialist-Led Acute Care (HiSLAC) study cited in the text.

Provenance and peer review Not commissioned; internally peer reviewed.

\section{REFERENCES}

1 Bell CM, Redelmeier DA. Mortality among patients admitted to hospitals on weekends as compared with weekdays. $N$ Engl J Med 2001;345:663-8.

2 Canadian Institute for Health Information. Weekend admissions and in-hospital mortality. [Report] June 2014. https://secure. cihi.ca/free_products/Mortality_Report_2014_en.pdf

3 Webb M. The weekend effect: a rapid review of the literature. 2011. http://www2.nphs.wales.nhs.uk:8080/healthservice qdtdocs.nsf/Main\%20Frameset?OpenFrameSet\&Frame= Right $\&$ Src $=\% 2$ Fhealthserviceqdtdocs.nsf\%2F61c1e930f91 21fd080256f2a004937ed\%2F7f113a4146140de1802578b 1002d7781\%3FOpenDocument\%26AutoFramed 
4 Cavallazzi R, Marik PE, Hirani A, et al. Association between time of admission to the ICU and mortality: a systematic review and metaanalysis. Chest 2010;138:68-75.

5 Sorita A, Ahmed A, Starr SR, et al. Off-hour presentation and outcomes in patients with acute ischemic stroke: a systematic review and meta-analysis. Eur J Intern Med 2014;25:394-400.

6 Sorita A, Ahmed A, Starr SR, et al. Off-hour presentation and outcomes in patients with acute myocardial infarction: systematic review and meta-analysis. BMJ 2014;348:f7393.

7 Ruiz M, et al. The Global Comparators Project: International comparison of 30-day in-hospital mortality by day of the week. BMJ Qual Saf 2015;24:492-504.

8 Bottle A, Middleton S, Kalkman CJ, et al. Global comparators project: international comparison of hospital outcomes using administrative data. Health Serv Res 2013;48(6 Pt 1):2081-100.

9 Perez Concha O, Gallego B, Hillman K, et al. Do variations in hospital mortality patterns after weekend admission reflect reduced quality of care or different patient cohorts? A population-based study. BMJ Qual Saf 2014;23:215-22.

10 Lilford R, Mohammed MA, Spiegelhalter D, et al. Use and misuse of process and outcome data in managing performance of acute medical care: avoiding institutional stigma. Lancet 2004;363:1147-54.

11 Ryan K, Levit K, Davis H. Characteristics of weekday and weekend hospital admissions. AHRQ Healthcare Cost and Utilization Project 2010: Statistical brief \#87.

12 Aujesky D, Jimenez D, Mor MK, et al. Weekend versus weekday admission and mortality after acute pulmonary embolism. Circulation 2009;119:962-8.
13 Mikulich O, Callaly E, Bennett K, et al. The increased mortality associated with a weekend emergency admission is due to increased illness severity and altered case-mix. Acute Med 2011;10:182-7.

14 Kostis WJ, Demissie K, Marcella SW, et al. Weekend versus weekday admission and mortality from myocardial infarction. N Engl J Med 2007;356:1099-109.

15 Girling AJ, Hofer TP, Wu J, et al. Case-mix adjusted hospital mortality is a poor proxy for preventable mortality: a modelling study. BMJ Qual Saf 2012;21:1052-6.

16 Percival F, Day N, Lambourne A, et al. An evaluation of consultant input into acute medical admissions management in England, Wales and Northern Ireland. 2010. https://www. rcplondon.ac.uk/sites/default/files/consultant-input-into-acutemedical-admissions-management-oct-2010.pdf

17 Buckley D, Bulger D. Trends and weekly and seasonal cycles in the rate of errors in the clinical management of hospitalized patients. Chronobiol Int 2012;29:947-54.

18 Attenello FJ, Wen T, Cen SY, et al. Incidence of "never events" among weekend admissions versus weekday admissions to US hospitals: national analysis. BMJ 2015;350:h1460.

19 HiSLAC. An evaluation of the impact of High-Intensity Specialist-Led Acute Care (HiSLAC) on emergency admissions to NHS hospitals at weekends. 2015. http://www.hislac.org/

20 Brown C, Lilford R. Evaluating service delivery interventions to enhance patient safety. BMJ 2008;337:a2746.

21 Yao GL, Novielli N, Manaseki-Holland S, et al. Evaluation of a predevelopment service delivery intervention: an application to improve clinical handovers. BMJ Qual Saf 2012;21(Suppl 1): i29-38. 